\title{
AAV Capsid Chimeras with Enhanced Infectivity reveal a core element in the AAV Genome critical for both Cell Transduction and Capsid Assembly
}

Lydia Viney ${ }^{a}$, Tilmann Bürckstümmer, Mario Mietzsch ${ }^{\mathrm{b}}$, Modassir Choudhry ${ }^{\mathrm{a}}$, Tom Henley ${ }^{{ }^{*}}$, Mavis Agbandje-McKenna ${ }^{b^{*}}$

alntima Bioscience, New York, USA; bepartment of Biochemistry and Molecular Biology, Center for Structural Biology, McKnight Brain Institute, University of Florida, Gainesville, 14 Florida, USA 


\section{ABSTRACT}

22 Adeno-associated viruses (AAV) have attracted significant attention in the field of gene and cell therapy due to highly effective delivery of therapeutic genes into human cells. The ability to generate recombinant $\mathrm{AAV}$ vectors compromised of unique or substituted protein sequences has led to the development of capsid variants with improved therapeutic properties. Seeking a novel AAV capable of enhanced transduction of human T cells for applications in immunotherapy, we have developed a unique capsid variant termed AAV $X$ Vivo (AAV-XV) that is a chimera of AAV12 VP1/2 sequences and the VP3 sequence of AAV6. This AAV chimera showed enhanced infection of human primary $\mathrm{T}$ cells and hematopoietic stem cells, and superiority over wildtype AAV6 for the genomic integration of DNA sequences either by AAV alone or in combination with CRISPR gene editing. AAV-XV demonstrated transduction efficiency equivalent to AAV6 at multiplicities of infection 2 logs lower, enabling T cell engineering at low AAV doses. Analyzing the protein coding sequence of $\mathrm{AAV}-\mathrm{XV}$ revealed disruptions within the assembly-activating protein (AAP) which likely accounted for observed lower virus yield. A series of genome alterations reverting the AAP sequence back to wildtype had a negative impact on the enhanced transduction seen with $A A V-V X$, indicating overlapping functions within this sequence for both viral assembly and effective $T$ cell transduction. Our findings show that $A A V-X V$ is highly efficient at $T$ cell engineering at low AAV dose and demonstrates the importance of AAP coding region in both viral particle assembly and cell infection. 
IMPORTANCE

50 A major hurdle to the therapeutic potential of AAV in gene therapy lies in achieving clinically

51 meaningful AAV doses, and secondarily, ability to manufacture commercially viable titers of

$52 \mathrm{AAV}$ to support this. By virtue of neutralizing antibodies against AAV that impede patient

53 repeat-dosing, the dose of AAV for in vivo gene delivery has been high, which has resulted

54 in unfortunate recent safety concerns and deaths in patients given higher-dose AAV gene

55 therapy. We have generated a new AAV variant possessing a unique combination of capsid

56 proteins for ex-vivo application termed AAV-XV, which delivers high levels of cell

57 transduction and gene delivery at a lower MOI. Furthermore, we demonstrate a novel

58 finding, and an important consideration for recombinant AAV design, that a region of the

59 AAV genome encoding the capsid protein and AAP gene is critical for both virus yield and

60 the enhancement of infection/transduction. 


\section{INTRODUCTION}

62 The Adeno-associated viruses (AAVs) are one of the most widely developed and actively studied vehicles for gene and cell therapy, and have shown remarkable promise in numerous clinical trials for multiple human disorders (1). As a vector for gene-delivery into human cells, these single-stranded DNA viruses show broad tropism, with multiple serotypes identified for transducing cells from many different tissue types (1). Their non-pathogenicity coupled with long-term transgene expression, makes AAV attractive as a therapeutic technology for in vivo gene therapy

(2). Despite broadly low innate immunogenicity, concerns over humoral immune responses against AAV capsids, observed in recent clinical trials, have been raised, particularly associated with high vector doses $(3,4)$. This in vivo limitation, as well as the high doses or multiplicities of infection (MOI) of virus required for sufficient cell transduction, and the need to expand the repertoire of transducible tissue types addressable with $\mathrm{AAV}$, is motivating further development of recombinant $\mathrm{AAV}$ technology.

There are 13 naturally occurring AAV serotypes and numerous AAV isolates (5), each with unique capsid viral protein (VP) sequences and transduction profiles in different tissues (6). As an example, AAV6 is consistently better than other serotypes in ex vivo transduction of human immune cells $(7,8)$. Each serotypes' distinct VP sequences assemble in strict $T=1$ icosahedral arrangement that enables packaging of the AAV genome into an

80 infectious virion (9). Novel variants of AAV are also being identified from sequencing experiments in different cell types, such as within CD34+ hematopoietic stem cells (10). Furthermore, uniquely engineered AAV vectors with enhanced transduction efficiencies have been developed (11) through capsid mutation by rational design $(12,13)$, directed evolution (14), or by combining different serotypes through capsid shuffling (15). Thus, combining sequences from divergent serotypes, or specific mutations of surface exposed capsid residues known to facilitate viral entry into cells, may be an effective route to improve the infectious properties of AAV. 
While AAV vector transduction can lead to high levels of transient transgene expression by episomal genomes, integration into the host genome typically occurs at very low frequency (16). The stable genomic integration of AAV donor vectors can be increased significantly via the combination of AAV vectors with CRISPR/Cas9 gene editing (17). A targeted double-strand break (DSB) introduced by Cas9 at a specific location within the genome can be effectively repaired with an AAV template designed with homology to the target locus, via the pathway of homology-directed repair (HDR) (17). This AAV + CRISPR combination approach has been effectively used by us and others to perform genetic engineering of difficult to target cell types such as primary human $T$ cells at levels of efficiency that are therapeutically relevant (18). However, despite the advances in AAV vector engineering, capsid evolution, and use of synergistic technologies such as CRISPR, high AAV dose MOls, typically $1 \times 10^{6}$ virus particles per cell, are still required for gene

100 delivery into cells being modified for either research or therapeutic applications (19). This 101 makes AAV a costly technology to deploy at scale for cell therapies, and as mentioned, may 102 preclude in vivo efficacy due to potentially toxic high doses required for gene therapy.

To address the above limitations, a unique AAV capsid variant with enhanced transduction of human $T$ cells was developed to improve the efficiency of ex vivo gene delivery. A series of capsid variants, were engineered, via rational design, by substituting the VP1 unique (VP1u) and VP1/2-common region sequences of AAV6 with those from divergent AAV serotypes such as AAV4, AAV5, AAV11, and AAV12 to create chimeric AAV6

108 vectors. Analysis of the resulting chimeras, for performance in transduction assays using 109 primary human T cells, found several variants that achieved levels of transduction 100 -fold 110 higher than wild-type AAV6 at similar MOI or 10- to 30-fold higher at 2 log lower doses. The 111 best performing variant was named AAV X-Vivo (AAV-XV). For this variant, an overlapping 112 region of the cap open reading frame (ORF) encoding VP2 and the Assembly-Activating 113 Protein (AAP), was observed to be critical for both optimal vector yield and efficient cellular 114 infectivity. Our data demonstrates that AAV-XV is highly efficient at cellular transduction and 
bioRxiv preprint doi: https://doi.org/10.1101/2020.10.13.337261; this version posted October 13,2020 . The copyright holder for this preprint (which was not certified by peer review) is the author/funder, who has granted bioRxiv a license to display the preprint in perpetuity. It is made available under aCC-BY-NC-ND 4.0 International license.

115 a broadly relevant recombinant vector for the ex-vivo engineering of human $\mathrm{T}$ cells for

116 immunotherapy and has potential as an efficient AAV for in vivo gene delivery at low doses.

117

118 
RESULTS

120

121 Generation of new chimeric AAV6 capsid variants. To engineer capsid variants with the

122 potential to transduce human T cells at high efficiencies, 7 chimeric AAV capsid sequences,

123 for which AAV6 provided the VP3, were generated (Supplementary Fig. S1). Each variant 124 incorporated sequence from the VP1u or VP1u+VP1/2 common regions of AAV4, AAV5, or

125 AAV12 or VP1u+VP1/2 common region of AAV11. The serotypes substituted represent the 126 most diverse based on the pairwise comparison of the VP1u and VP1/2 common regions 127 from AAV1 through AAV13 with AAV6 (Supplementary Fig. S1), with the intent to create 128 maximum diversity in the resulting chimeras. A multiple sequence alignment between the 129 selected serotypes and AAV6 showed conservation of functional regions including a PLA2 130 motif (20), a calcium binding motif (21), and basic residue clusters that serve as nuclear 131 localization sequences (NLS) (22), although positioning of the latter was different for AAV5

132 (Fig. 1A). This alignment showed a higher sequence identity for the VP1u region compared 133 to the VP1/2 common region (Supplementary Fig. S1). Recombinant AAV vectors utilizing 134 wild-type AAV6 and the 7 variant capsids (Fig. 1B), packaging a donor template with a 135 NanoLuc luciferase gene, were produced and evaluated for their performance as ex vivo gene-delivery vectors in human T and stem cells.

Chimeric AAV6 capsid variants outperform wildtype as donors for CRISPR genomic

139 integration in human $\mathbf{T}$ and stem cells at low MOI. To assess the impact of the VP 140 sequence substitutions into AAV6 on infectivity and genomic integration efficiencies, CD3+ T 141 cells were electroporated with Cas9 mRNA and a sgRNA targeting the AAVS1 locus, 142 followed by infection with the rAAV chimeras (Fig. 1B). The rAAV vectors delivered a 143 NanoLuc expression cassette flanked by homology arms allowing targeted integration at the 144 AAVS1 locus (23) (Fig. 2A). An MOI titration of these variants monitored 7- and 14-days post 145 infection and CRISPR transfection showed 3 of the 7 chimeras (variants 2, 4, and 5), with 146 significantly enhancement transduction and genomic integration of the targeting construct 
147 (1.5 to 2 logs) compared to wild-type AAV6 (Fig. 2B). Interestingly, at a low MOI of $1 \times 10^{4}$

148 genome copies per cell (gc/cell), the best variant, 5, named AAV-XV, achieved luciferase

149 levels that required a 100-fold higher $\mathrm{MOI}$ of wild-type AAV6 vectors $\left(1 \times 10^{6}\right)$. Variants 2, 4,

150 and 5 possess the VP1u+VP1/2 common region of AAV4, and AAV11, and AAV12,

151 respectively (Fig. 1B), and unlike AAV5 and AAV6, contain an additional basic cluster (Fig.

$1521 \mathrm{~A})$. In contrast, the variants containing only the VP1u region of AAV4, and AAV11, and

153 AAV12 did not display enhanced transduction and genomic integration suggesting that their

154 VP1/2 common region is the determinant of this phenotype (Fig. 1B and 2B). Notably, the

155 variant with substituted VP1+VP1/2 common region of AAV5 (variant 3) did not enhance

156 transduction and was arguably the worse performing vector (Fig. 2B).

157 Further analysis of the 3 best performing capsid variants in both human cytotoxic

158 CD8+ T cells and CD34+ HSCs confirmed higher transduction efficiencies and luciferase targeting to the AAVS1 genomic site of up to 2 logs (Fig. 3A). This enhanced performance

160 was also observed in the absence of a CRISPR-mediated DSB, with variant 5 showing a 1

161 log increase in transduction efficiency over AAV6 when measured up to 21 days post 162 infection (Fig. 3B). Again, this variant achieved similar levels of $\mathrm{T}$ cell transduction at the 163 lower $\mathrm{MOI}$ of $1 \times 10^{4} \mathrm{gc} / \mathrm{cell}$ as AAV6 at $\mathrm{MOI}$ of $1 \times 10^{6} \mathrm{gc} / \mathrm{cell}$. AAV-XV thus demonstrated superior infectivity and transduction performance at low doses compared to the wild-type AAV6 sequence from which it was derived.

167 Transduction efficiency was inversely correlated with variant yield. Several of the 168 capsid chimeras routinely produced lower than wild-type AAV6 viral titers from packaging 169 cell lines, while other variants showed no detectible reduction (Fig. 4A). When the T cell 170 transduction performance of each capsid variant was compared to their viral titer, the 3 best 171 performing variants, 2, 4, and 5, were on average 3 logs lower than wild-type AAV6.

172 Comparison of the viral titer of the purified wild-type AAV6 and these three capsid variants 173 by quantitative PCR after DNase I treatment, showed significantly lower titers, 2 to 3 logs, for 174 the three variants (Fig. 4B). Quantification of viral capsids by ELISA, using an antibody 
175 recognizing an epitope present on AAV6 and all capsid variants, confirmed that all variants

176 showed a reduced number of viral particles, also at 2 to 3 logs less than wild-type AAV6

177 (Fig. 4B). This observation indicated that the capsid substitutions in variants 2, 4, and 5

178 impacted either capsid assembly or the stability of assembled viral particles.

179 Attempts to improve variant 5 capsid yield by either optimizing AAV vector 180 transfection method, varying the ratio of AAV helper and AAV donor plasmid transfected, or

181 by extending duration of cell transfection prior to harvesting from the supernatant, failed to

182 demonstrate any detectible increase in viral particle titer (Fig. 4C). All conditions tested

183 resulted in similar vector yield as measured by qPCR (not shown) and average purified

184 particle yield as measured by ELISA (Fig. 4C). Variant 5 capsid yield remained at $\sim 2$ to 3

185 logs lower than for wild-type AAV6. Toward understanding the mechanistic reason for this

186 impairment in particle assembly, the region of the AAV6 capsid sequence altered in these capsid chimera variants were investigated.

The AAP sequence has an impact on the transduction efficiency of AAV6 capsid

chimeras. Given the importance of AAP for AAV capsid assembly $(24,25)$, the alterations in the sequences of each of the capsid variants within their cap ORF coding for AAP was analyzed. In addition to the VP sequence, the AAP sequence was changed relative to wildtype AAV6 in 4 of the 7 capsid chimeras containing the VP1u+VP1/2 common region (Fig.

$1945 \mathrm{~A}$, and data not shown), including the 3 variants showing enhanced transduction at low MOls (Fig. 1A and 4A). Previous studies identified the importance of the AAP N-terminus in capsid stability and assembly $(26,27)$. Thus, to restore a potentially lost AAP function for variant 5 , a series of substitution mutants were generated in which the AAP-12 residues equivalent to AAP-6 positions aa13 to aa27 were gradually reverted back to AAV6 (Fig. 5A).

199 A complete rescue of viral titer, to levels of wild-type AAV6, was achieved by reintroducing AAP-6 (Variant 5.1) (Fig. 5B). This observation confirmed the hypothesis that variant 5 has an assembly defect due to compromised AAP function. Approximately 1 log rescue was 
corresponding to AAP-6 residues aa21 to aa27, the start of the AAP-6 hydrophobic region, were reintroduced to the chimera (Variant 5.3). The remaining substitution variants could not effectively rescue the assembly defect (Fig. 5B).

We further demonstrated that co-transfection with a functional full-length CMV promoter-driven AAP-6 gene into the producer cells, in trans, along with the AAV-XV Rep/Cap and donor vector, rescued titer by up to 1.5 logs (Fig. 5C). The AAP gene with the natural CTG start codon (leucine) present in the AAP-6 sequence was insufficient to provide this rescue, while substitution to an ATG start codon to code a methionine, produced the titer

211 rescue (Fig. 5C). This likely reflects the requirement to have a canonical start signal for

212 robust translation initiation and expression of AAP when present within an expression 213 plasmid in HEK293 packaging cells.

Finally, the AAV12VP1/2-AAV6VP3 variant that was fully (variant 5.1) or partially (variant 5.3) rescued by AAP-6 sequence restoration were assessed for T-cell transduction and genomic integration of the luciferase gene when co-delivered with CRISPR (Fig. 5D).

217 Variant 5.1, in which AAP-6 residues aa13 to aa27 had been reverted to AAV6, gave the 218 same level of transduction as AAV6 at all MOls compared, and had thus lost any 219 enhancement in infectivity or transduction (Fig. 5D). In contrast, variant 5.3, in which only aa21 to aa27 of AAP-6 were reintroduced, maintained some level of superiority over AAV6, resulting in $2 \log$ higher transduction at $\mathrm{MOI}$ of $1 \times 10^{5} \mathrm{gc} / \mathrm{cell}$ and $1 \log$ higher transduction at the lowest $\mathrm{MOI}$ tested of $1 \times 10^{4} \mathrm{gc} / \mathrm{cell}$. Variant 5 maintained superiority to AAV6 at $\mathrm{MOI}$ $1 \times 10^{4} \mathrm{gc} / \mathrm{ml}$ (Fig. 5D).

Variant 5 vectors, generated with the Met-AAP-6 co-transfection construct, resulted in levels of T cell transduction approximately 2 logs higher than AAV6, and is almost as high as the level observed for variant 5 that is AAP-disrupted (Fig. 5E). The Leu-AAP-6 cotransfection produced sample shows transduction that is $\sim 1$ log higher than wild-type AAV6 (Fig. 5E). Collectively these data demonstrate that a modified AAP sequence in the capsid chimera variants can restore vector production at the expense of transduction efficiency at

230 lower MOls. However, co-transfection with AAP in trans during vector production also 
231 partially restores packaging ( $>1 \log$ ) and with minimal impact on transduction at low MOI

232 (Fig. 5C and E).

\section{DISCUSSION}

235 Recombinant AAV vectors hold great promise as a gene delivery vehicle $(1,2)$. Despite demonstrated clinical efficacy reported for several indications, limitations remain that impede the broader applicability of the AAV technology for efficient and persistent gene delivery to many cell types. The low frequency genomic integration of AAV had previously made the genetic engineering of cells a difficult and laborious task that required the use of drug selection genes or fluorescent markers to select the small number of cells successfully modified with the AAV donor (28). The advent of designer nuclease technologies and the combination of CRISPR gene editing with AAV, enabled substantially higher levels of targeted genome integration of AAV donors and opened up the ability to genetically modify cell types such as primary human T cells for therapeutic applications $(17,18)$. However, even with these advances in technology, high $\mathrm{MOI}$ doses of AAV are still required to reach sufficient levels of transgene integration. Despite optimized production procedures, high dose requirements of $\mathrm{AAV}$ are expensive and limit scaling to clinical manufacture. Perhaps even more importantly, recent evidence of immune responses directed towards large quantities of AAV administered in vivo to patients in gene therapies highlights the potential toxicity issues associated with high AAV doses $(3,4)$. In an effort to expand and improve the transduction efficiency and genetic engineering potential of $A A V$, our chimeric $A A V$ variant design approach, combining VPs from several divergent serotypes, has generated an AAV, AAV-XV, with enhanced transduction characteristics for human $T$ cells that address the requirement for high $\mathrm{MOI}$. AAV6 is considered the best serotype for the transduction of $\mathrm{T}$ cells, and thus served as a starting point to evaluate the impact of VP1 and VP2 swapping

(Fig. 1) in an attempt to identify modifications that further improved this T cell tropism (19). 
259 AAV6 with equal efficiency. Both as a donor template in combination with CRISPR gene 260 editing, and as a method for AAV-mediated homologous recombination in the absence of 261 targeted genomic cleavage, superior transduction and stable gene delivery was observed for 262 AAV-XV when compared to AAV6 (Fig. 2 and 3). While the mechanism for this enhanced transduction is not yet clear, the enhanced transduction efficiency requires the C-terminal amino acid sequences of the AAV12 VP1/2 common region. The VP1/2 common region as well as the N-terminus of VP3 are believed to be located in the interior of the capsid and becomes externalized to the capsid surface upon acidification of the endosome during cellular trafficking (29). Thus, the observed enhanced efficiency of infection is likely to be a post-entry effect due to improved interaction with trafficking receptors/effectors. Furthermore, this region has been described as structurally highly flexible (30) and no significant structural differences are predicted (http://original.disprot.org/) by substituting AAV6 to AAV12 sequences.

What is clear from the data is that the modification of the AAV6 capsid sequence to 273 incorporate the VP1/2 of AAV12, results in a change of the amino acid sequence for the 274 overlapping AAP. This is likely the reason for the lower titer yield for the AAV vectors with 275 the variants compared to the wild-type AAV6 given the well characterized assemblypromoting activity of AAP and identification of mutations within this sequence that reduces the interaction of AAP with the capsid, impairing its ability to promoter particle assembly (24-

$27826,31,32)$. The critical region affected in the AAV-XV variant lies within a hydrophobic region of AAP and aa13-27 appear particularly important for stability and assembly functions (26). The data demonstrates that the defect can also be partially rescued by restoring aa2127 to AAP-6, supporting the theory that these capsid changes impaired AAP-mediated capsid assembly.

The overlapping ORF encoding the VPs and AAP add complexity to the rational design of capsid variants (24). This was evident in the chimeras generated in this study in which VP changes resulted in AAP modification, and low vector yield. However, the ability to 286 transduce cultured human T cells at several log orders lower MOls to achieve efficient 
genomic integration of AAV donor DNA requires far less virus to be manufactured, achieving

a balance between potency and yield. In conclusion, our data has shown that AAV capsid chimeras generated by VP protein combinations from divergent serotypes is an effective approach for generating novel AAV variants with unique and enhanced functional properties for cellular transduction. Careful consideration of the precise sequence changes is important given the overlapping nature of AAV ORFs and particular care is needed to avoid

293 detrimental modifications to the AAP or MAAP protein (33). The AAV-XV capsid chimera,

294 AAP rescued or not, shows the useful property of highly efficient transduction of cultured human cells at 100-fold lower MOls compared to the parental AAV6. AAV-XV thus has valuable properties for low-dose gene delivery, enhancing the safety profile of AAV vectors administered in vivo to avoid the toxicity that can occur with current AAV-based therapies at high dose.

\section{METHODS}

AAV variant design and plasmid generation. AAV variants were designed by first extracting the sequences of the VP1 1 and VP1/2 common region from AAV1 through AAV13 (included AAVrh.10) and performing a pairwise alignment of each serotype to AAV6 using Clustal Omega (https://www.ebi.ac.uk/Tools/msa/clustalo/). The pairwise identity and similarity for each serotype sequence was compared and serotypes with the lowest identities within the VP1u and VP1/2 common region, (serotypes AAV4, AAV5, AAV11, and AAV12) were used to generate the chimeric sequences with the AAV6 VP3. At this point these chimeras were called variant 1 to 7 . DNA encoding the chimera regions were generated by DNA synthesis (GenScript Biotech) and subcloned into the AAV6 RepCap plasmid to replace the AAV6 sequence (Plasmid Factory).

A gene targeting vector to measure genome integration via AAV homologous

313 recombination, was constructed by flanking the NanoLuc luciferase gene under the control

314 of the CMV promoter, with $1 \mathrm{~kb}$ sequences homologous to a transcriptionally active intronic 
region within the human AAVS1 locus. This AAVS1 luciferase donor contained AAV2 ITRs

316 for packaging of the single-stranded vector into chimeric AAV6 particles, and the ampicillin

317 resistance gene.

319 AAV production, purification, and quantification of the genomic titer. Recombinant

AAV6 variants were produced by ViGene Biosciences by triple transfection of adherent growing HEK293 cells with the AAV6 variant RepCap plasmids, helper plasmid, and NanoLuc luciferase donor plasmid using polyethylenimine. The transfected cells were harvested $72 \mathrm{~h}$ post transfection, pelleted, and subjected to three freeze-thaw cycles to release the AAV vectors from the cells. Vectors released into the grows medium during the $72 \mathrm{~h}$ incubation period were recovered by addition of polyethylene glycol (PEG) to a final concentration of $8.2 \%(\mathrm{w} / \mathrm{v})$ and subsequent precipitation. The rAAVs from the cell pellet and the PEG precipitate were combined and treated with benzonase for $30 \mathrm{~min}$ to $2 \mathrm{~h}$. The raw lysate was clarified by centrifugation and the supernatant purified by iodixanol gradient ultracentrifugation, as previously described (34), using a Beckman VTI 50 rotor at 48,000 rpm for $2 \mathrm{~h}$. The genome-containing capsids were extracted from the $40 \%$ iodixanol fraction which buffer-exchanged and concentrated using an Amicon® Ultracel 100 kDa cut-off concentrator column (Millipore).

The packaged genome titers were determined by quantitative PCR (qPCR) using Sybr Green stain, with primers directed to the AAV2 inverted terminal repeat regions. The physical particle titer was determined by an AAV6 titration ELISA (Progen, PRAAV6) according to the manufacturer's instructions, that recognizes a conformational epitope present on AAV6 and all other capsid variants tested here, but does not detect unassembled capsid proteins.

340 T cell transduction and luciferase assay. Primary human CD3+ and CD8+ T cells were

341 isolated from unfractionated PBMCs using the EasySep Human T cell Isolation Kit and

342 Human CD8 T cell isolation kit with RapidSpheres (Stemcell Technologies). Mobilized 
human primary CD34+ cells from Peripheral Blood (were obtained from Caltag Medsystems,

344 Buckingham, UK). Both T cells and CD34+ HSCs were cultured in X-Vivo 15 media (Lonza) supplemented with 10\% human serum AB (Merck Sigma-Aldrich), $300 \mathrm{IU} / \mathrm{ml} \mathrm{IL-2,} \mathrm{and} 5$ $\mathrm{ng} / \mathrm{mL} \mathrm{IL}-7$ and IL-15 (Peprotech) at $37^{\circ} \mathrm{C}$ and $5 \% \mathrm{CO}_{2}$.

For CRISPR + AAV treatments, $2 \times 10^{5} \mathrm{~T}$ cells were first stimulated using antiCD3/CD28 dynabeads (Invitrogen) in complete T cell media for $48 \mathrm{~h}$ prior to electroporation.

349 T cells were electroporated with 15 ug Cas9 mRNA (TriLink) and 10 ug AAVS1 specific sgRNA using the Neon electroporator $\left(3 \times 10^{5}\right.$ in $10 \mathrm{ul}$ Neon tip) and pulse conditions $1400 \mathrm{~V}$, $10 \mathrm{~ms}, 3$ pulses. Electroporated T cells were recovered in T cell media for $2 \mathrm{~h}$ before addition of purified $\mathrm{AAV}$ vectors to the media at $\mathrm{MOI}$ ranging from 200 to $1 \times 10^{6}$ viral particles. The volume of each virus sample was adjusted by diluting in PBS, so each treatment received equivalent volumes, compensating for the lower concentrated vector samples. Media was replaced after $24 \mathrm{~h}$ with fresh complete T cell media, and again every 2 to $3 \mathrm{~d}$. For quantification of $\mathrm{T}$ cell transduction, luciferase activity of the transduced $\mathrm{T}$ cells was measured at 7, 14, and $21 \mathrm{~d}$ post-infection. T cells were harvested, and firefly luciferase analyzed using the Dual-Glo Luciferase assay kit (Promega) according to the manufacturer's instructions. Luminescence was measured using a PHERAstar microplate reader (BMG Labtech).

Investigation the role of AAP on AAV6 variant production. For experiments investigating the impact of co-expressing wildtype AAP during viral packaging, AAV production protocols were modified to include polyethylenimine co-transfection of an AAP-6 expression plasmid (ORF under the control of the CMV promoter, synthesized by GenScript Biotech), the variant 5 AAV cap plasmid, and an adenoviral helper plasmid into HEK293 in equivalent amounts, along with the AAVS1-Nano Luciferase targeting vector. Constructs expressing the AAP gene with either the native Leucine start codon, or a substituted Methionine start codon were tested. Virus produced using expression of AAP in trans were purified as described above. 
For experiments investigating the reversion back to wild-type AAP-6 within the AAP modified in the variants, additional vectors were designed and synthesized with combinations of amino acid substitutions with residues 13-27 of the AAP-6 sequence. Virus preparations packaging the AAVS1-Nano Luciferase construct using each AAP-modified variant were generated and evaluated for transduction in human T cells as described above.

Figure 1. Design of recombinant AAV6 capsid variants. (A) Alignment of the VP1u and VP1/2 common region sequences of AAV4, AAV5, AAV6, AAV11, and AAV12 showing conservation of functionally important motifs. (B) Schematic diagram of the AAV2 Rep and the AAV serotypes contributing VP1, VP2, and VP3 to the AAV6 variants. The approximate positions of promoters are indicated. A serotype color key is provided.

Figure 2. Transduction efficiency and CRISPR-mediated genomic integration of AAV6 capsid variants in human T cells. (A) Schematic of the AAV6 targeting vector for the integration of a luciferase expression cassette into the PPP1R12C (AAVS1) genomic locus. Diagram indicates the relative position of CRISPR-mediated genomic cleavage and the resulting modified locus upon homology-directed repair with the AAV6-luciferase vector. (B) MOI dose-titration of wild-type AAV6 and chimeric AAV6 capsid variants measuring CRISPR-mediated genomic integration of a luciferase reporter gene in human CD3+ $\mathrm{T}$ cells at 7 and $14 \mathrm{~d}$ post-infection. Statistical significance was determined by a one-way ANOVA test for multiple comparisons, ${ }^{* * * *} \mathrm{P}<0.0001$.

Figure 3. Low MOI transduction of human CD8+ T cells and HSCs by AAV6 capsid variants. (A) Comparison of CRISPR-mediated genomic integration by wild-type AAV6 and

396 the top three performing AAV6 capsid variants at low MOI $\left(1 \times 10^{4} /\right.$ cell) in primary CD8 T cells (left) and human CD34+ HSCs (right) (B) Comparison of transduction efficiency of 


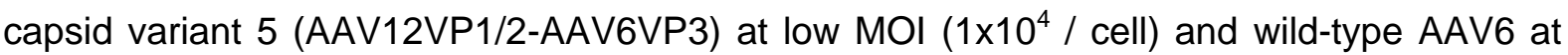
high $\mathrm{MOI}\left(1 \times 10^{6} /\right.$ cell) in CD8 T cells over a 3-week period in the absence of CRISPR gene editing. Statistical significance was determined by an unpaired T test, or one-way ANOVA test for multiple comparisons, ${ }^{*} \mathrm{P}<0.05,{ }^{* \star} \mathrm{P}<0.01,{ }^{* *} \mathrm{P}<0.001 \quad{ }^{* * \star *} \mathrm{P}<0.0001$.

402

Figure 4. AAV6 capsid variants are defective for capsid assembly. (A) Viral packaging titers and T cell luciferase transduction values for AAV6 capsid variants and the impact of the chimeric capsid sequences on the coding protein sequence of AAP. High rate of transduction correlates with low titer. A heat map coloring is used to indicate the magnitude of yield and transduction. Viral preparations for AAV6 and variants 2, 4, and 5 were produced 3 times; variant 1, 2 times and variants 3,6 , and 7 were produced once. (B) Viral packaging titers from purified AAV particle yield measured by $\mathrm{qPCR}$ and ELISA. Both are reduced for variants 2, 4, and 5 compared to wild-type AAV6. (C) Modifying transfection conditions has no effect on AAV6 variant capsid yield. Varying the transfection reagent, the ratio of AAV helper plasmid transfected or the virus harvest time post-transfection, did not lead to any increase in viral genome or particle titer (as measured above) for variant 5 . Wildtype AAV6 and variant 5 genome and particle titers were measured from purified particles. Statistical significance was determined by an unpaired T test, or one-way ANOVA test for multiple comparisons of results in triplicate or duplicate replicas, ${ }^{* \star *} \mathrm{P}<0.001,{ }^{* \star * *} \mathrm{P}<0.0001$. GC, Genome copies; RLU, Relative light units.

Figure 5. The AAP sequence plays a role in gene expression in addition to capsid assembly. (A) Amino acid sequence alignment of the AAP sequences at the VP2/VP3 boundary for wild-type AAV6 and capsid variant 5 modifications. The wild-type AAV6 sequence is at the top, capsid variant 5 AAP with changes reverting to the wild-type sequence at amino acids 13-27 (outlined by the dashed-line box) of AAP, are 5.1 to 5.6. (B)

424 Genome titers for wild-type AAV6, capsid variant 5, and 5.1 to 5.6 with altered AAP sequences. (C) Genome titer of wild-type AAV6, capsid variant 5 alone and with co- 
transfection of two forms of the full-length AAP construct in the packaging cells, one starting with a Methionine and the other a Leucine. (D) AAP sequence alterations play a role on transduction of primary CD8+ $T$ cells. Viruses are as in (C). Complete correction of amino acids 13-27, as in variant 5.1 , decreases $\mathrm{T}$ cell transduction levels, whereas partially corrected 5.3 along with the original variant 5 surpass wild-type AAV6 at low MOI. (E) T cell transduction by variant 5 packaged by co-transfection of the AAP expression constructs (as in D). Statistical significance was determined by an unpaired T test, or one-way ANOVA test for multiple comparisons of results in triplicate or duplicate replicas, ${ }^{*} P<0.05,{ }^{* \star} P<0.01$, ${ }^{* * *} \mathrm{P}<0.001 \quad{ }^{* * * *} \mathrm{P}<0.0001$

\section{Supplementary}

S1. Amino acid capsid sequence alignment of the AAV6 chimera variants with wild-type

AAV6. Below a table of the amino acid sequence identities of AAV4, $-5,-11,-12$ and the variants to the AAV6 VP1, VP1u, and VP1/2 common region is given, respectively.

\section{Conflicts of Interest}

M.A.-M. is a consultant for, and this work is funded by Intima Bioscience. Intima Bioscience has patents filed based on the findings described herein. The authors declare no competing interests.

\section{REFERENCES}

1. Wang D, Tai PWL, Gao G. 2019. Adeno-associated virus vector as a platform for gene therapy delivery. Nat Rev Drug Discov 18:358-378.

2. Naso MF, Tomkowicz B, Perry WL, 3rd, Strohl WR. 2017. Adeno-Associated Virus (AAV) as a Vector for Gene Therapy. BioDrugs 31:317-334.

3. Ronzitti G, Gross DA, Mingozzi F. 2020. Human Immune Responses to AdenoAssociated Virus (AAV) Vectors. Front Immunol 11:670.

4. Srivastava A. 2020. AAV Vectors: Are They Safe? Hum Gene Ther 31:697-699.

5. Gao G, Vandenberghe LH, Alvira MR, Lu Y, Calcedo R, Zhou X, Wilson JM. 2004. Clades of Adeno-associated viruses are widely disseminated in human tissues. J Virol 78:6381-8.

6. Srivastava A. 2016. In vivo tissue-tropism of adeno-associated viral vectors. Curr Opin Virol 21:75-80.

7. Song L, Kauss MA, Kopin E, Chandra M, Ul-Hasan T, Miller E, Jayandharan GR, Rivers AE, Aslanidi GV, Ling C, Li B, Ma W, Li X, Andino LM, Zhong L, Tarantal 

AF, Yoder MC, Wong KK, Jr., Tan M, Chatterjee S, Srivastava A. 2013. Optimizing the transduction efficiency of capsid-modified AAV6 serotype vectors in primary human hematopoietic stem cells in vitro and in a xenograft mouse model in vivo. Cytotherapy 15:986-98.

8. Yang H, Qing K, Keeler GD, Yin L, Mietzsch M, Ling C, Hoffman BE, AgbandjeMcKenna M, Tan M, Wang W, Srivastava A. 2020. Enhanced Transduction of Human Hematopoietic Stem Cells by AAV6 Vectors: Implications in Gene Therapy and Genome Editing. Mol Ther Nucleic Acids 20:451-458.

9. Mietzsch M, Penzes JJ, Agbandje-McKenna M. 2019. Twenty-Five Years of Structural Parvovirology. Viruses 11.

10. Smith LJ, Ul-Hasan T, Carvaines SK, Van Vliet K, Yang E, Wong KK, Jr., Agbandje-McKenna M, Chatterjee S. 2014. Gene transfer properties and structural modeling of human stem cell-derived AAV. Mol Ther 22:1625-34.

11. Li C, Samulski RJ. 2020. Engineering adeno-associated virus vectors for gene therapy. Nat Rev Genet 21:255-272.

12. Burg M, Rosebrough C, Drouin LM, Bennett A, Mietzsch M, Chipman P, McKenna R, Sousa D, Potter M, Byrne B, Jude Samulski R, Agbandje-McKenna M. 2018. Atomic structure of a rationally engineered gene delivery vector, AAV2.5. J Struct Biol 203:236-241.

13. Bowles DE, McPhee SW, Li C, Gray SJ, Samulski JJ, Camp AS, Li J, Wang B, Monahan PE, Rabinowitz JE, Grieger JC, Govindasamy L, Agbandje-McKenna M, Xiao X, Samulski RJ. 2012. Phase 1 gene therapy for Duchenne muscular dystrophy using a translational optimized AAV vector. Mol Ther 20:443-55.

14. Davidsson $M$, Wang $G$, Aldrin-Kirk P, Cardoso $T$, Nolbrant S, Hartnor $M$, Mudannayake J, Parmar M, Björklund T. 2019. A systematic capsid evolution approach performed in vivo for the design of AAV vectors with tailored properties and tropism. Proceedings of the National Academy of Sciences 116:27053-27062.

15. Li W, Asokan A, Wu Z, Van Dyke T, DiPrimio N, Johnson JS, Govindaswamy L, Agbandje-McKenna M, Leichtle S, Eugene Redmond D, Jr., McCown TJ, Petermann KB, Sharpless NE, Samulski RJ. 2008. Engineering and Selection of Shuffled AAV Genomes: A New Strategy for Producing Targeted Biological Nanoparticles. Mol Ther 16:1252-1260.

16. Colella P, Ronzitti G, Mingozzi F. 2018. Emerging Issues in AAV-Mediated In Vivo Gene Therapy. Mol Ther Methods Clin Dev 8:87-104.

17. Gaj T, Staahl BT, Rodrigues GMC, Limsirichai P, Ekman FK, Doudna JA, Schaffer DV. 2017. Targeted gene knock-in by homology-directed genome editing using Cas 9 ribonucleoprotein and AAV donor delivery. Nucleic Acids Res 45:e98.

18. Bak RO, Porteus MH. 2017. CRISPR-Mediated Integration of Large Gene Cassettes Using AAV Donor Vectors. Cell Rep 20:750-756.

19. Wang J, DeClercq JJ, Hayward SB, Li PW, Shivak DA, Gregory PD, Lee G, Holmes MC. 2016. Highly efficient homology-driven genome editing in human T cells by combining zinc-finger nuclease mRNA and AAV6 donor delivery. Nucleic Acids Res 44:e30.

20. Girod A, Wobus CE, Zadori Z, Ried M, Leike K, Tijssen P, Kleinschmidt JA, Hallek M. 2002. The VP1 capsid protein of adeno-associated virus type 2 is carrying a phospholipase A2 domain required for virus infectivity. J Gen Virol 83:973-8.

21. Canaan S, Zadori Z, Ghomashchi F, Bollinger J, Sadilek M, Moreau ME, Tijssen P, Gelb MH. 2004. Interfacial enzymology of parvovirus phospholipases A2. J Biol Chem 279:14502-8. 
22. Grieger JC, Snowdy S, Samulski RJ. 2006. Separate basic region motifs within the adeno-associated virus capsid proteins are essential for infectivity and assembly. J Virol 80:5199-210.

23. Oceguera-Yanez F, Kim SI, Matsumoto T, Tan GW, Xiang L, Hatani T, Kondo T, Ikeya M, Yoshida Y, Inoue H, Woltjen K. 2016. Engineering the AAVS1 locus for consistent and scalable transgene expression in human iPSCs and their differentiated derivatives. Methods 101:43-55.

24. Sonntag F, Kother K, Schmidt K, Weghofer M, Raupp C, Nieto K, Kuck A, Gerlach B, Bottcher B, Muller OJ, Lux K, Horer M, Kleinschmidt JA. 2011. The assemblyactivating protein promotes capsid assembly of different adeno-associated virus serotypes. J Virol 85:12686-97.

25. Sonntag F, Schmidt K, Kleinschmidt JA. 2010. A viral assembly factor promotes AAV2 capsid formation in the nucleolus. Proceedings of the National Academy of Sciences of the United States of America 107:10220-5.

26. Naumer M, Sonntag F, Schmidt K, Nieto K, Panke C, Davey NE, Popa-Wagner R, Kleinschmidt JA. 2012. Properties of the adeno-associated virus assembly-activating protein. J Virol 86:13038-48.

27. Tse LV, Moller-Tank S, Meganck RM, Asokan A. 2018. Mapping and Engineering Functional Domains of the Assembly-Activating Protein of Adeno-associated Viruses. J Virol 92.

28. Khan IF, Hirata RK, Russell DW. 2011. AAV-mediated gene targeting methods for human cells. Nat Protoc 6:482-501.

29. Kronenberg S, Bottcher B, von der Lieth CW, Bleker S, Kleinschmidt JA. 2005. A conformational change in the adeno-associated virus type 2 capsid leads to the exposure of hidden VP1 N termini. J Virol 79:5296-303.

30. Venkatakrishnan B, Yarbrough J, Domsic J, Bennett A, Bothner B, Kozyreva OG, Samulski RJ, Muzyczka N, McKenna R, Agbandje-McKenna M. 2013. Structure and dynamics of adeno-associated virus serotype 1 VP1-unique N-terminal domain and its role in capsid trafficking. J Virol 87:4974-84.

31. Grosse S, Penaud-Budloo M, Herrmann AK, Borner K, Fakhiri J, Laketa V, Kramer C, Wiedtke E, Gunkel M, Menard L, Ayuso E, Grimm D. 2017. Relevance of Assembly-Activating Protein for Adeno-associated Virus Vector Production and Capsid Protein Stability in Mammalian and Insect Cells. J Virol 91.

32. Herrmann AK, Große S, Börner K, Krämer C, Wiedtke E, Gunkel M, Grimm D. 2019. Impact of the Assembly-Activating Protein on Molecular Evolution of Synthetic Adeno-Associated Virus Capsids. Hum Gene Ther 30:21-35.

33. Ogden PJ, Kelsic ED, Sinai S, Church GM. 2019. Comprehensive AAV capsid fitness landscape reveals a viral gene and enables machine-guided design. Science 366:11391143.

34. Zolotukhin S, Byrne BJ, Mason E, Zolotukhin I, Potter M, Chesnut K, Summerford C, Samulski RJ, Muzyczka N. 1999. Recombinant adeno-associated virus purification using novel methods improves infectious titer and yield. Gene Ther 6:973-85. 


$$
\begin{aligned}
& \stackrel{\text { VP1 }}{\longmapsto} \\
& 10 \\
& 20 \\
& 30 \\
& 40 \\
& \text { binding }
\end{aligned}
$$

AAV6 MAADGYLPDWLEDNLSEGIREWWDLKPGAPKPKANQQKQDDGRGLVLPGYKYLGPFNGLDKGEPVNAADA AAV4 MTDGYLPDWLEDNLSEGVREWWALQPGAPKPKANQQHQDNARGLVLPGYKYLGPGNGLDKGEPVNAADA AAV5 MSFVDHPPDWLEE-VGEGLREFLGLEAGPPKPKPNQQHQDQARGLVLPGYNYLGPGNGLDRGEPVNRADE AAV11 MAADGYLPDWLEDNLSEGIREWWDLKPGAPKPKANQQKQDDGRGLVLPGYKYLGPFNGLDKGEPVNAADA AAV12 MAADGYLPDWLEDNLSEGIREWWALKPGAPQPKANQQHQDNGRGLVLPGYKYLGPFNGLDKGEPVNEADA

$$
{\stackrel{\text { motif }}{\text { PLA }_{2}}}_{80}
$$

$110 \quad 120 \stackrel{\text { BR1 }}{ }$ 130

$\stackrel{\text { VP2 }}{\stackrel{140}{\longrightarrow}}$

AAV6 AALEHDKAYDQQLKAGDNPYLRYNHADAEFQERLQEDTSFGGNLGRAVFQAKKRVLEPFGLVEEGAKTAP AAV4 AALEHDKAYDQQLKAGDNPYLKYNHADAEFQQRLQGDTSFGGNLGRAVFQAKKRVLEPLGLVEQAGETAP AAV5 VAREHDISYNEQLEAGDNPYLKYNHADAEFQEKLADDTSFGGNLGKAVFQAKKRVLEPFGLVEEGAKTAP AAV11 AALEHDKAYDQQLKAGDNPYLRYNHADAEFQERLQEDTSFGGNLGRAVFQAKKRVLEPLGLVEEGAKTAP AAV12 AALEHDKAYDKQLEQGDNPYLKYNHADAEFQQRLATDTSFGGNLGRAVFQAKKRILEPLGLVEEGVKTAP

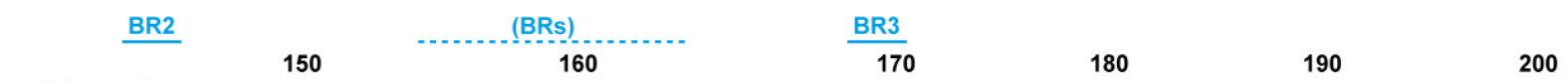
AAV6 GKKRPVEQSPQE---PDSSSGIGKTGQ-----QPAKKRLNFGQTGDSESVPDPQPLGEPPATPAAVGPTT AAV4 GKKRPLIESPQQ---PDSSTGIGKKGK-----QPAKKKLVFEDETGAG---DGPPEGSTSGA AAV5 TGKRIDDHFP-----KRKKARTEEDSK--------PSTSSDAEAGPSGS----QQLQIPAQPASSLGADT AAV11 GKKRPLES-PQE---PDSSSGIGKKGK-----QPARKRLNFEEDTGAG---DGPPEGSDTSA AAV12 GKKRPLEKTPNRPTNPDSGKAPAKKKQKDGEPADSARRTLDFEDSGAG---DGPPEGSSSGE

\section{Figure 1b}

AAV6

Variant 1: AAV5VP1u-AAV6VP2/3

Variant 2: AAV4VP1/2-AAV6VP3

Variant 3: AAV5VP1/2-AAV6VP3

Variant 4: AAV11VP1/2-AAV6VP3

Variant 5: AAV12VP1/2-AAV6VP3

Variant 6: AAV4VP1u-AAV6VP2/3

Variant 7: AAV12VP1u-AAV6VP2/3
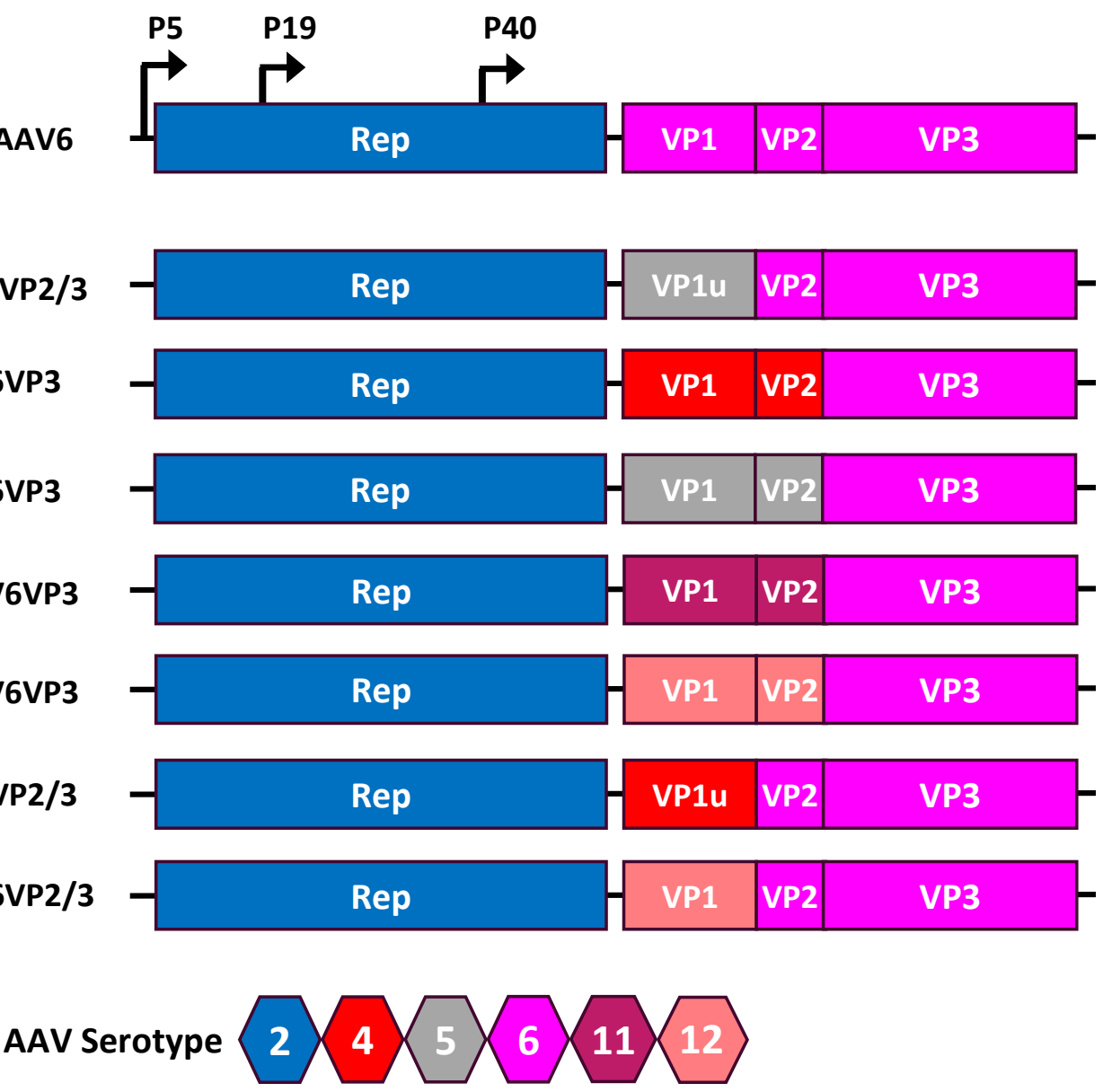


\section{Figure 2a}

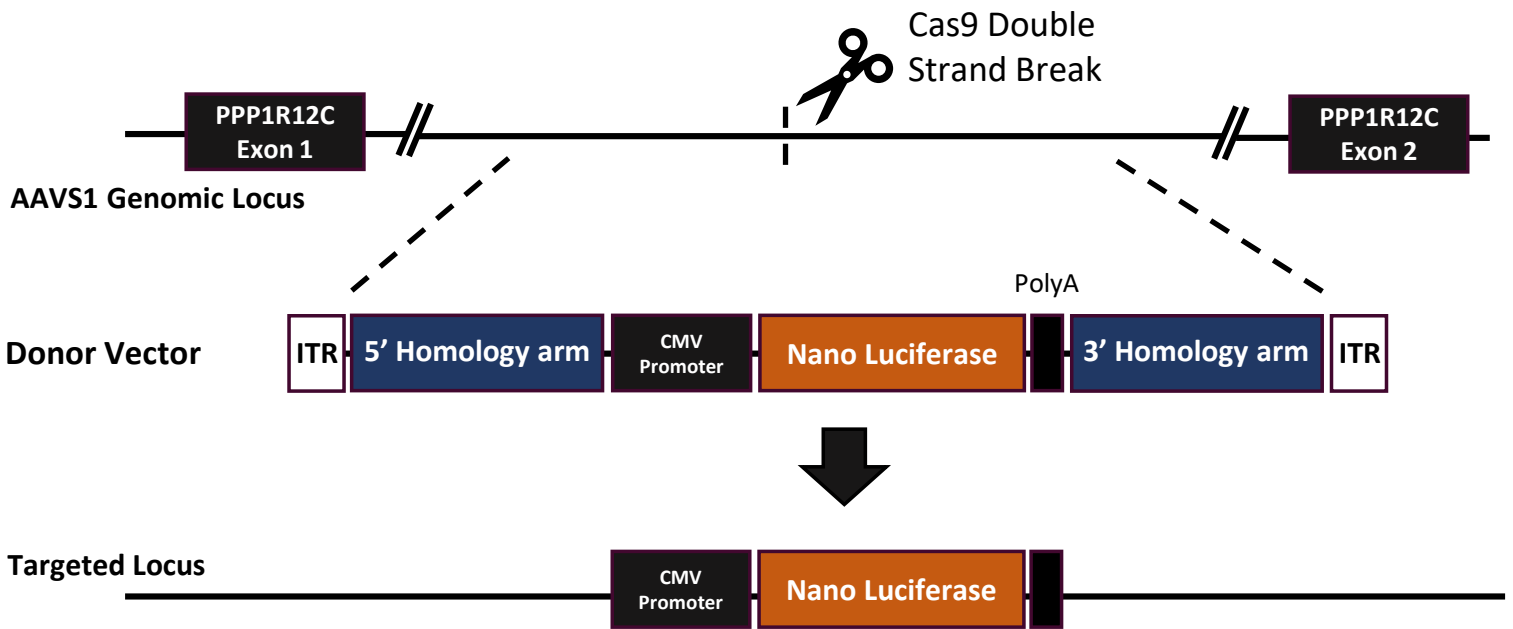

Figure 2b

Transduction efficiency at Day 7

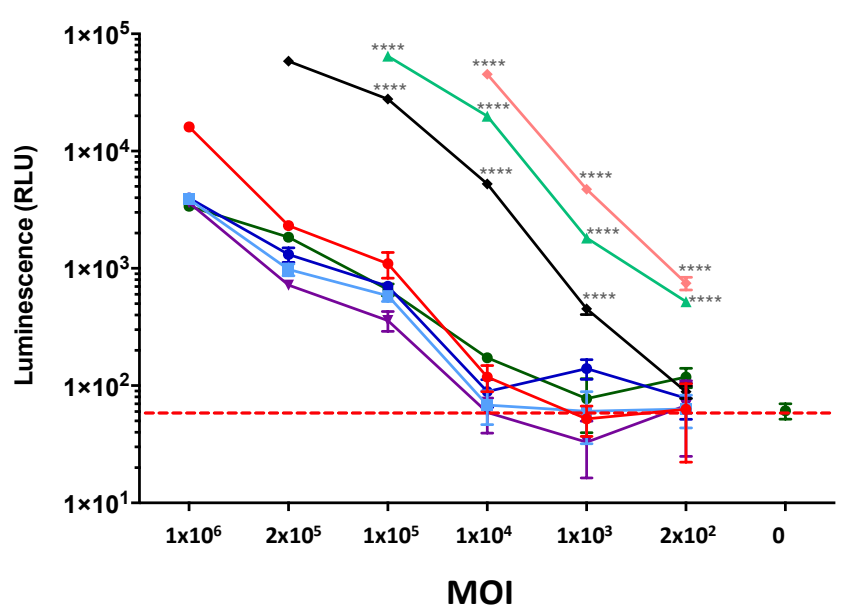

Transduction efficiency at Day 14

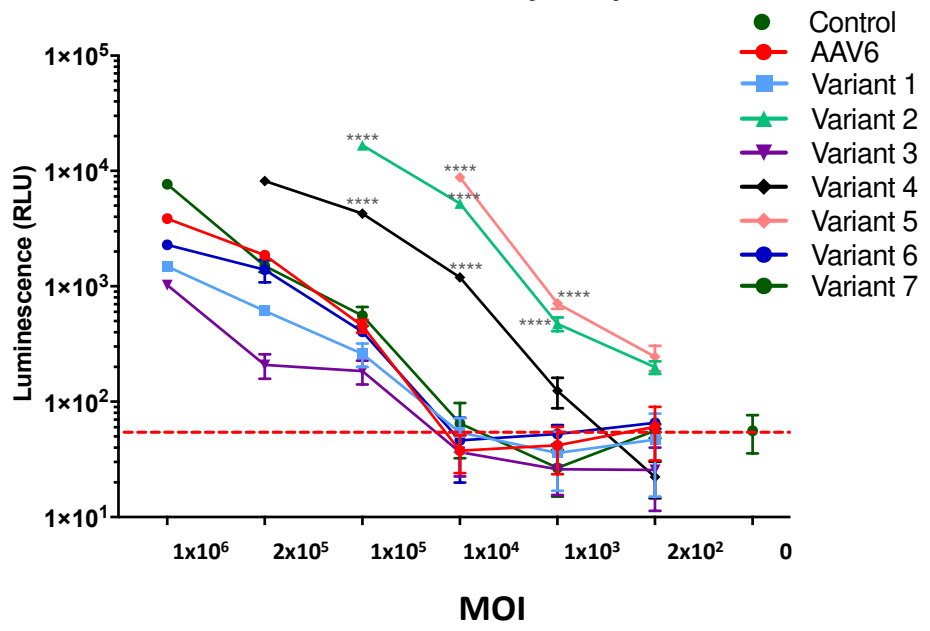


Figure 3a
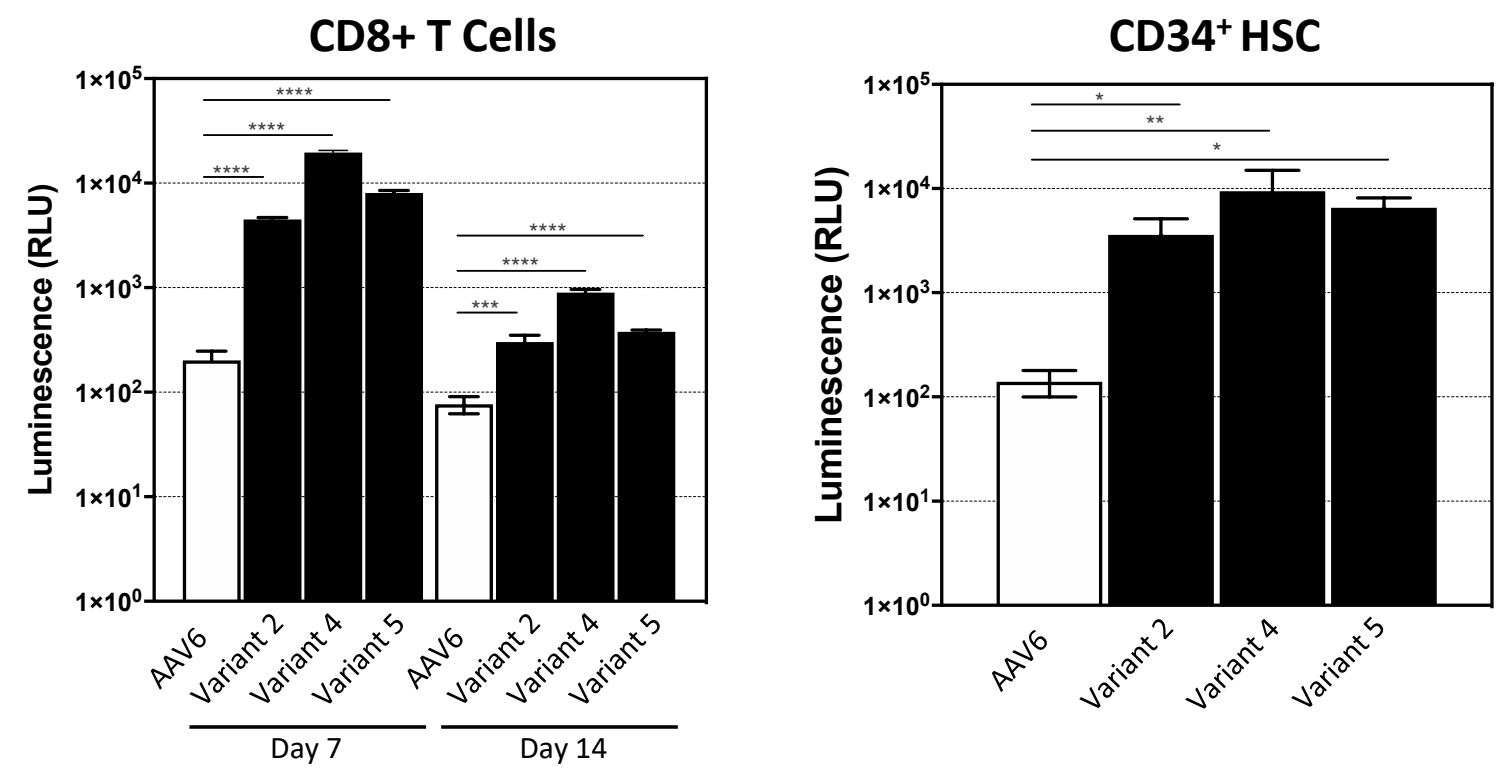

Figure $3 b$
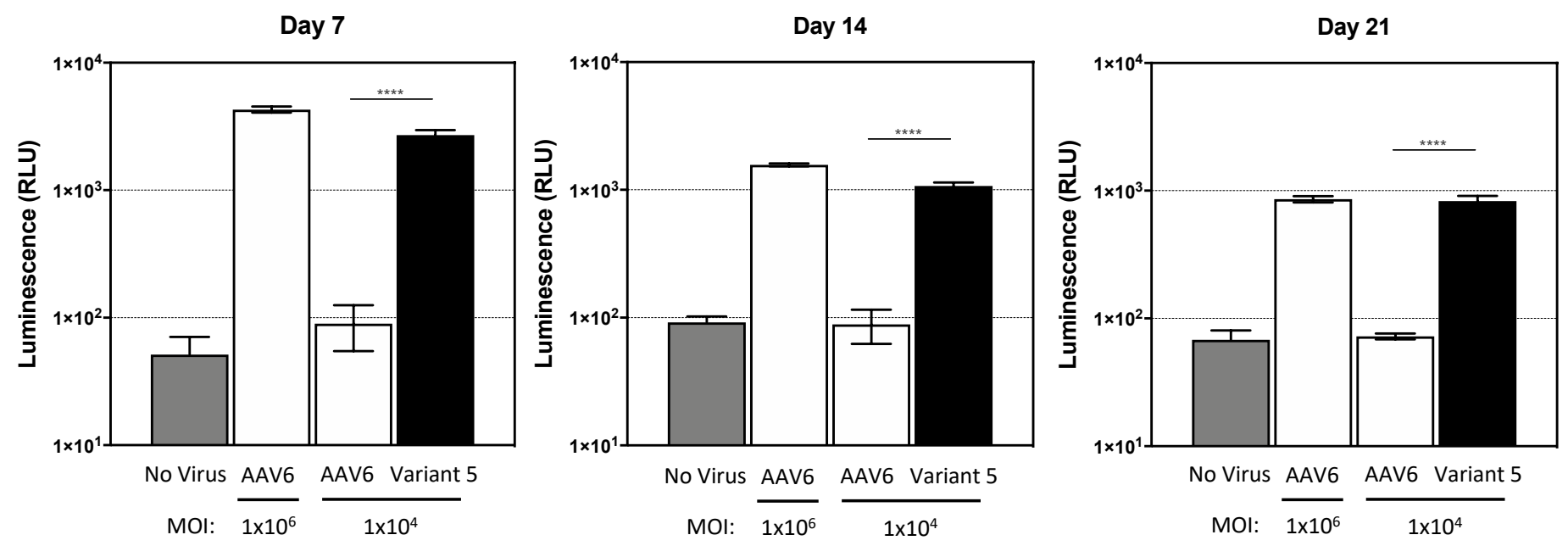


\section{Figure 4a}

\begin{tabular}{|c|c|c|c|c|}
\hline Variant Chimera & $\begin{array}{c}\text { Virus Titre } \\
\left(\times 10^{10} \mathrm{GC} / \mathrm{ml}\right)\end{array}$ & $\begin{array}{l}\text { Transduction Efficiency } \\
\text { (Luminescence RLU) }\end{array}$ & $\begin{array}{l}\text { AAP Chimeric } \\
\text { Serotype }\end{array}$ & $\begin{array}{l}\text { Impact on AAF } \\
\text { ORF }\end{array}$ \\
\hline AAV6 & $1161.33 \pm 764.16$ & $32.33 \pm 4.93$ & AAV6 & None \\
\hline Variant 1: AAV5VP1u-AAV6VP2/3 & $2995 \pm 784.89$ & $74.00 \pm 18.03$ & AAV6 & None \\
\hline Variant 2: AAV4VP1/2-AAV6VP3 & $4.49 \pm 1.97$ & $5699 \pm 395.76$ & AAV4/AAV6 & Modified \\
\hline Variant 3: AAV5VP1/2-AAV6VP3 & 1760.00 & $44.00 \pm 10.44$ & AAV5/AAV6 & Modified \\
\hline Variant 4: AAV11VP1/2-AAV6VP3 & $4.62 \pm 3.39$ & $779.67 \pm 359.35$ & AAV11/AAV6 & Modified \\
\hline Variant 5: AAV12VP1/2-AAV6VP3 & $0.42 \pm 0.03$ & $17141.67 \pm 14258.84$ & AAV12/AAV6 & Modified \\
\hline Variant 6: AAV4VP1u-AAV6VP2/3 & 2530.00 & $48.00 \pm 2.83$ & AAV6 & None \\
\hline Variant 7: AAV12VP1u-AAV6VP2/3 & 4340.00 & $59.00 \pm 8.49$ & AAV6 & None \\
\hline
\end{tabular}

\section{Figure 4b}
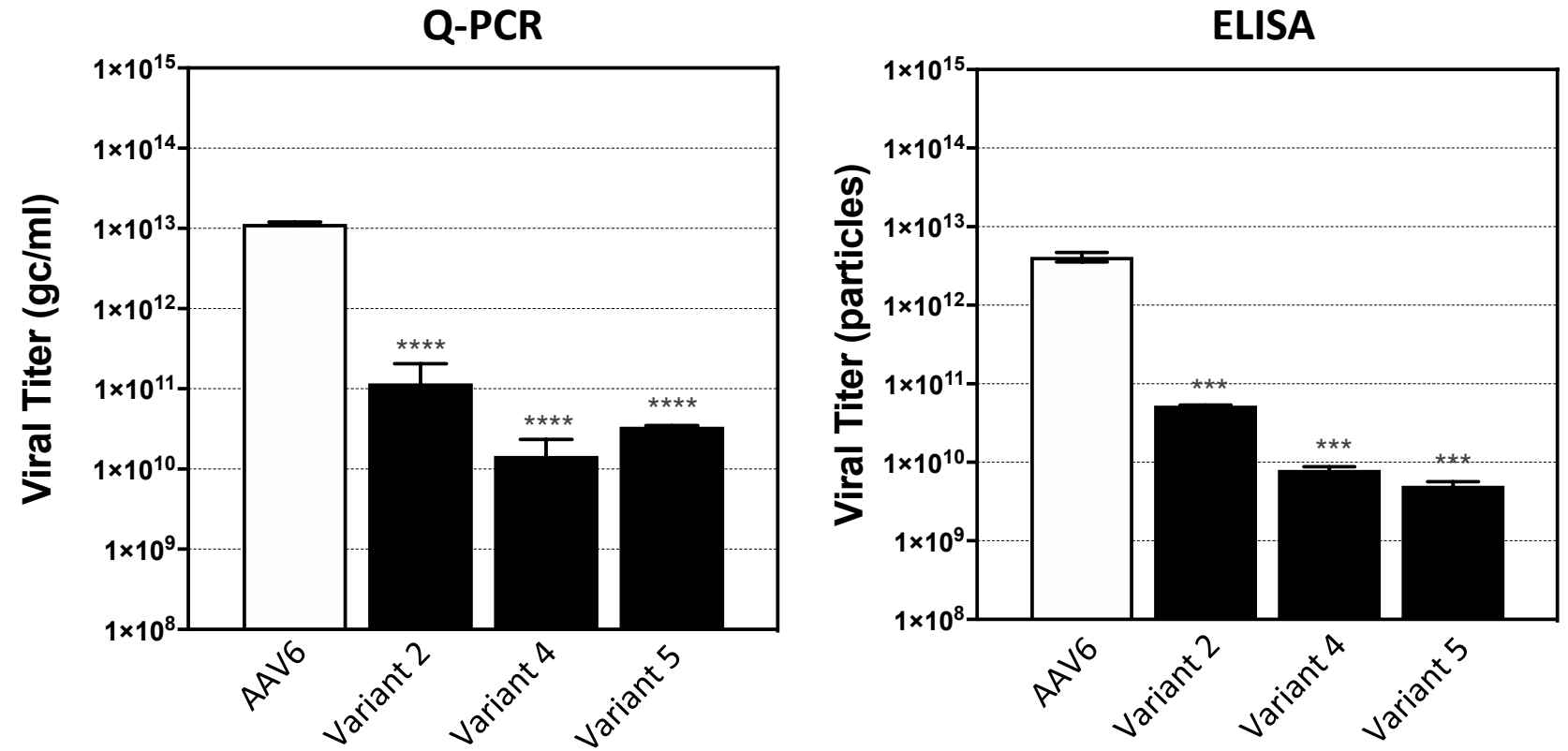

Figure 4c

ELISA

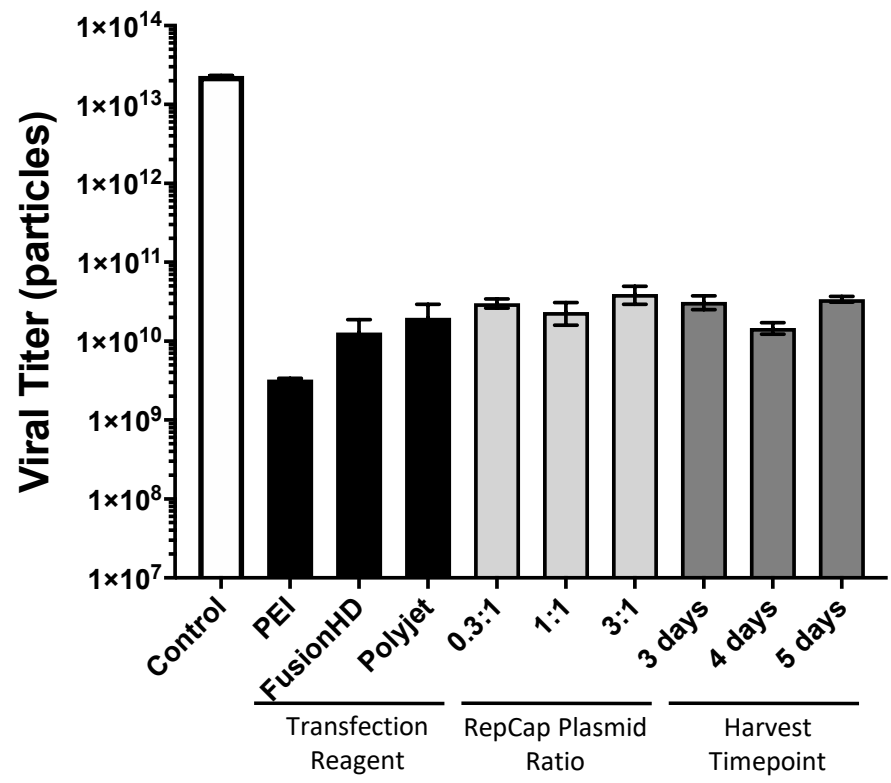


Figure 5a

AAP-6 LATQSQSPTHNL'SENLQQPPLLWDLLQWNLAVA AAP Variant 5.1 AAP Variant 5.2 AAP Variant 5.3 AAP Variant 5.4 AAP Variant 5.5 AAP Variant 5.6 AAP Variant 5

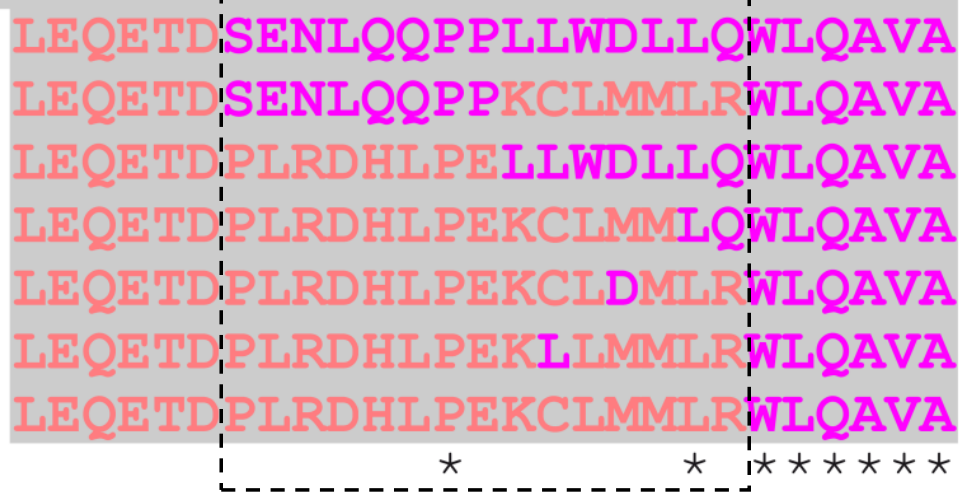

Figure 5b

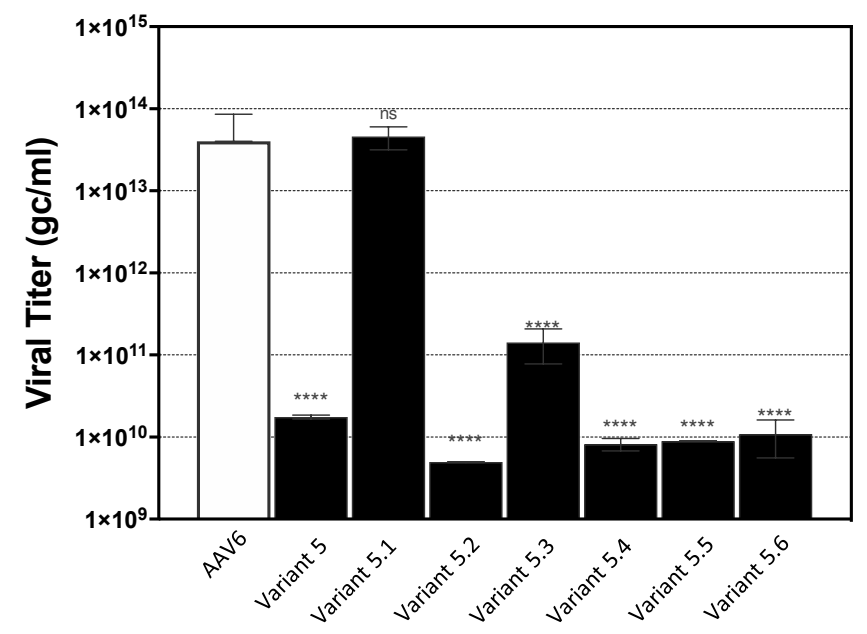

Figure 5d

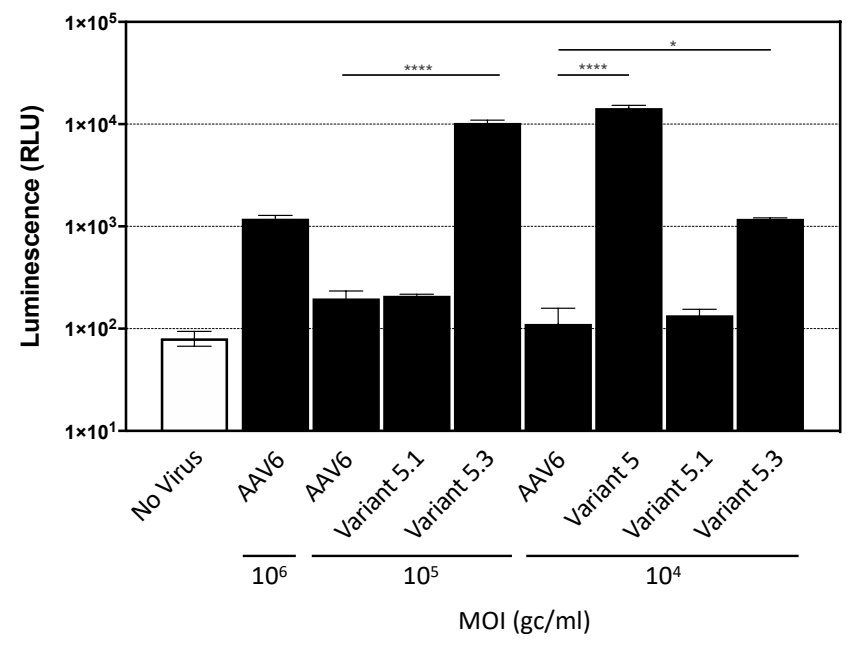

Figure 5c

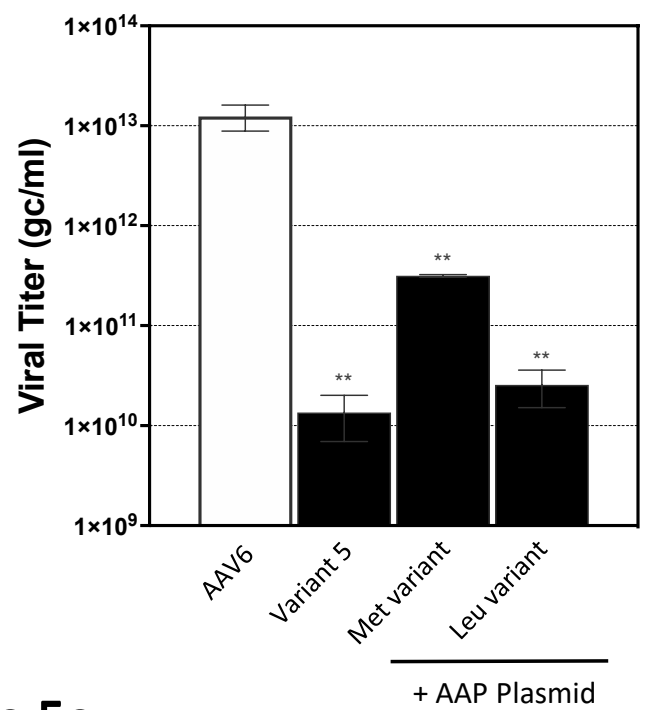

Figure 5e

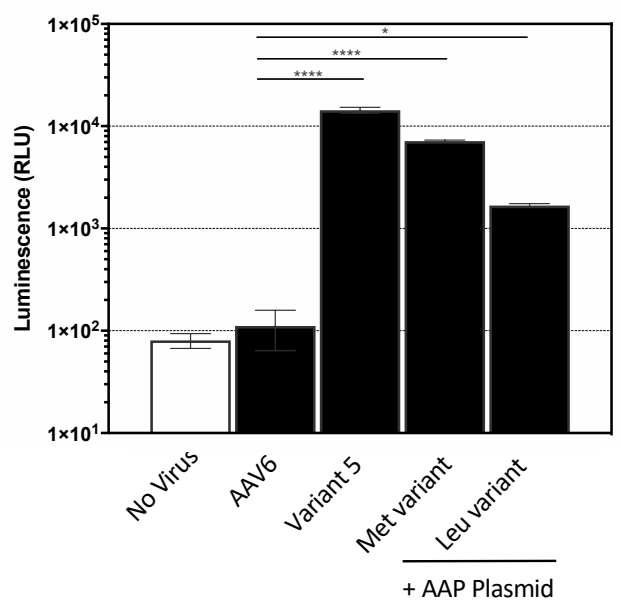

